\title{
Development of 3D human kidney model using Urine-Derived Epithelial Cells and HUVECs
}

Aatif Basher, MS1 1,2, William Goggins, BS ${ }^{1}$, Erika Gramelspacher, BS ${ }^{1}$, Julia Walsh', Mark H. Kaplan, $\mathrm{PhD}^{2}$, Wenjun Zhang, $\mathrm{PhD}^{1}$, Bruce Molitoris, $\mathrm{MD}^{3}$, Mervin Yoder, $\mathrm{MD}^{2}$, Burcin Ekser, MD, $\mathrm{PhD}^{1}$, Ping Li, $\mathrm{PhD}^{1}$

(1) Transplant Surgery, Department of Surgery, Indiana University School of Medicine

(2) Department of Pediatrics, Indiana University School of Medicine

(3) Department of Nephrology, Indiana University School of Medicine

Background and Hypothesis: Development of realistic, scaffold-free 3D organoids/tubuloids such as renal-like tissue, can serve as models for study of renal drug toxicity/screening, tissue repair, and renal disease. Previous studies utilized urine-derived epithelial cells (UDEpCs) to create viable tubuloid structures suspended in 3D Matrigel, displaying renal epithelial gene expression and functionality. Alternatively, renal proximal tubule epithelial cells (PTECs) with HUVECs were co-cultured in a monolayer to improve the model. Therefore, we hypothesized that the addition of HUVECs will help to establish and optimize growth conditions of UDEpCs in a 3D environment.

Experimental design: UDEpCs were isolated and co-cultured with tdTomatoHUVECs in Matrigel with different combinations of growth media. Cell growth and endothelial cell tube formation were examined daily. PTECs and tdTomatoHUVECs were also used to make spheroids in low-binding plates. Real-time PCR analysis was performed to examine gene expression for epithelial markers (ABCC4, PAX8), an endothelial marker (PECAM1), and TGF- $\beta$ in spheroids.

Results: A single cell-derived colony was isolated from human urine and was double positive for CD10 and CD13, two renal PTEC markers. Co-culture of UDEpCs and tdTomato-HUVECs in Matrigel dramatically increased the growth, survival, and tubuloid formation of both cell types, for up to 12 days. Total RNA from spheroids showed a decline over time in all conditions. Gene expression was decreased in co-culture conditions, but patterns of gene expression were maintained.

Conclusions and Potential Impact: Future studies will examine gene expression in Matrigel cells, further optimize conditions for growth of UDEpCs, and perform functional assays, such as brush border enzyme activity. Improved methods to develop 3D kidney organoids/tubuloids will produce more accurate models for renal function studies. Developing a functional model mimicking in vivo processes can lead to new applications for creating novel therapies and treatment strategies. 